\title{
Appendiceal Colic in Children: Is It a True Surgical Entity?
}

\author{
Jason Marone*, Domingo Alvear \\ Department of Surgery, Pinnacle Health, Harrisburg, USA \\ Email: *jmarone@pinnaclehealth.org
}

Received December 15, 2011; revised January 30, 2012; accepted February 19, 2012

\begin{abstract}
Background/Purpose: For decades children have been crowding the offices of Pediatricians' and Pediatric Surgeons with a common complaint of unexplained recurrent right lower quadrant pain. For years the topic of appendiceal colic as a cause for this recurrent abdominal pain has remain controversial. This paper highlights the experience of a single surgeon over a 5-year period using an algorithmic approach to diagnose and treat children with appendiceal colic. Methods: This is a retrospective review of 31 consecutive cases of patients experiencing recurrent unexplained right lower quadrant abdominal pain for greater than three weeks. All patients were evaluated by a single surgeon at the same institution. The patients meeting the criteria for appendiceal colic had an elective open appendectomy. The data included was compiled by a retrospective chart review from Jan. 1, 2006 to Dec. 31, 2010. Results: Twelve percent or 31 of the 238 elective appendectomies done between Jan. 2006 to Dec. 2010 where done for appendiceal colic. Sixteen were females and 15 where males ranging in age from 5 - 17 years of age. Average duration of symptoms was 3 weeks to 5 years. All patients had previous work up most of which where inconclusive for an organic cause. All patients presented with colic and McBurney's point tenderness. On pathological examination the presence of a fecalith, inspissated stool or fibrosis were the most common findings. A normal appendix was observed in $14 \%$ of the cases. $100 \%$ of the patients were symptom free with three months of operation. Conclusion: Appendiceal colic proves to be true in a small subset of patients presenting with unexplained right lower quadrant abdominal pain. A high index of suspicion coupled with a thorough history and physical exam is needed to identify these patients. Classically they will present with recurrent right lower abdominal pain at McBurney's. In our experience an elective appendectomy has been curative.
\end{abstract}

Keywords: Natural Asset; Financial Value; Neural Network

\section{Introduction}

Recurrent abdominal pain in the pediatric population is a common symptom that continues to frustrate pediatricians and pediatric surgeons. An organic cause is found in less than ten percent of these cases. The concept of appendiceal colic was first described by Robertson in 1934 [1]. Since this time authors such as Bowers, et al. have reported both saline distention and faradically induced appendiceal contractions in awake patients, thus confirming that the appendix as a likely cause for pain [2]. In the early 1980, Schisgall described the concept of inspissated cast of stool as a cause for appendiceal colic [3]. He stated the pain was caused by the appendix contracting as it tried to extrude the stool from the appendiceal lumen. This article was further supported by Stevenson who demonstrated in his article in 1999 that an elective appendectomy in patients with appendiceal colic is curative [4]. Many articles have since been published to describe the natural history, presentation, histological findings, and operative management of appendiceal colic. But the question of weather appendiceal colic is a true surgical entity, remains controversial.

\footnotetext{
*Corresponding author.
}

\section{Methods and Materials}

A retrospective chart review was conducted from January 1, 2006 to December 31, 2010. 238 elective appendectomies were performed, of these 31 patients underwent and elective appendectomy for the diagnosis if appendiceal colic. Diagnostic criteria was based on a thorough history and physical that demonstrated McBurney's point tenderness during painful episode, symptoms persisted for greater than three weeks, and having greater than three episodes. The majority of these patients also had pain exacerbation with eating or physical activity. All patients had a prior radiographic work up with either an abdominal ultrasound or an abdominal CT scan for their symptoms, and all workups were negative. A single surgeon at a single institution evaluated all patients. The patients diagnosed with appendiceal colic, all underwent an open appendectomy. Patient follow was conducted at two and four weeks post operation.

\section{Results}

Of the 31 patients, 16 were girls (50.16\%), and 15 were boys $(49.84 \%)$. The median age was 13 years with age 
range of 5 - 17 years. The mean duration of symptoms was 3.5 weeks with a range of 3 weeks to five years. The majority of patients had symptoms for three to four weeks, but five patients had symptoms for greater than one year.

The most common symptoms was recurrent colic and McBurney's point tenderness which were present in all patients as demonstrated in Table 1. 27 of the 31 patients (87\%) had 4 or more episodes prior to our evaluation. The remaining four patients had 3 episodes of recurrent right lower quadrant abdominal pain prior to evaluation. Thirty eight percent of the patients experienced aggravation of symptoms with physical activity. While 25 of the $31(80 \%)$, patients complained of nausea.

26 patients (83\%) had undergone at least one evaluation by their pediatrician or emergency room. All patients had routine blood work done all of which where normal. One patient had a CRP elevated to 3.6. 22/31 (70\%) patients underwent a right lower quadrant abdominal ultrasound, 17/31 (54\%) had an abdominal CT scan, and 13/31 (41\%) had both studies done to evaluate the recurrent right lower quadrant abdominal pain. In all case the imaging did not reveal an organic cause. An inconclusive GI workup was performed in 4/31 (12.9\%) patients. Eleven of the thirty-one patients (35\%), had documented missed days of school.

The pathological findings are outlined in Table 2. In our series the most common pathological finding was a fecalith obstruction of the appendiceal lumen. Fibrosis and inspissated cast of stool were the second most common findings (Figures 1-3). Of note all patients with symptoms longer than one year demonstrated fibrosis. The appendix was found to normal in $16 \%$ of the cases.

Table 1. Signs and symptoms.

\begin{tabular}{ccc}
\hline Signs and symptoms & Number of patients $(\mathrm{n}=31)$ & Percentage \\
\hline Colic & 31 & 100 \\
Nausea & 25 & 80 \\
Vomiting & 9 & 29 \\
Loose stools & 5 & 16 \\
Aggravated by activity & 12 & 38 \\
McBurney's point tenderness & 31 & 100 \\
\hline
\end{tabular}

Table 2. Pathology findings.

\begin{tabular}{ccc}
\hline Pathology & Number of patients $(\mathrm{n}=31)$ & Percentage \\
\hline Fecalith & 11 & 35 \\
Kink & 2 & 6.5 \\
Inspissated cast & 6 & 19 \\
Fibrosis & 6 & 19 \\
Enterobius & 3 & 9.6 \\
Focal inflammation & 5 & 16 \\
Lymphoid hyperplasia & 4 & 12.9 \\
Normal & 5 & 16 \\
\hline
\end{tabular}

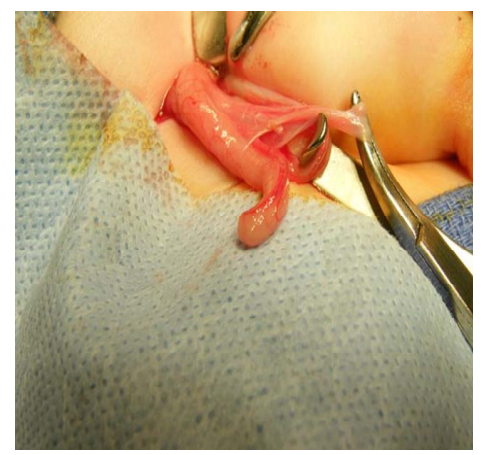

Figure 1. Fibrosis.

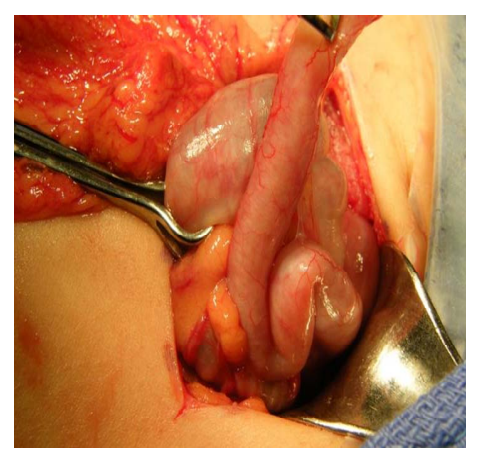

Figure 2. Kink.

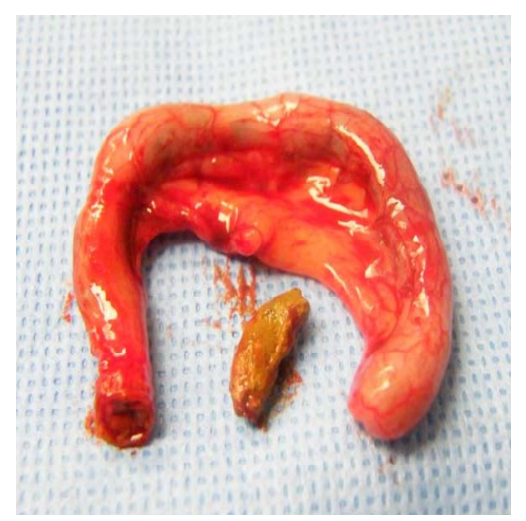

Figure 3. Fecalith.

The patients in our study group had been evaluated at 2 and 4 weeks post surgery. All patients were free of symptoms at four weeks. Those patient experiencing pain with exercise were able to return to their sport of choice without eliciting pain. An elective appendectomy is a viable choice for a small subset of patients meeting the criteria for appendiceal colic. Elective appendectomy was $100 \%$ effective in our experience, and has been documented throughout the literature to be $95 \%$ effective.

\section{Discussion}

For decades pediatricians and pediatric surgeons alike have evaluated countless patients for a common complaint of recurrent right lower quadrant abdominal pain. 
Often these patients' treatments and workups are drawn out over months to years without resolution of symptoms or defining a cause of their pain. These patients are often given the diagnosis of chronic appendicitis, chronic constipation, irritable bowel syndrome, or even a diagnosis of gynecological origin [5]. The pediatric literature suggests nonoperative management. In a study produced by the Mayo Clinic in which nonoperative management was employed in children presenting with recurrent RLQ abdominal pain, the average time for resolution of symptoms was 3.5 years. Apley estimated that two-thirds of the children treated nonoperatively continued to have symptoms [6]. It wasn't until the 1937 that D. E. Robertson described concept of appendiceal colic as a cause for recurrent RLQ abdominal pain [3]. In the early 19th century an elective appendectomy for recurrent abdominal pain carried a significantly high failure rate and mortality rate. This coupled with the lack of consistency in defining appendiceal colic has lead to ongoing controversy.

Appendceal colic has a slight female predominance and most often found in children in their early teens. Most authors will agree that colic is severe crampy abdominal pain associated with spasms of a hollow viscous. Among the pediatric population there are other types of colic that present, such as biliary colic, renal colic, GI colic, and adnexal colic, which need to be rolled out. It is said that only $10 \%$ of the time will an organic cause be found for recurrent right lower quadrant abdominal pain [3]. A high index of suspicion for appendiceal colic is needed and thus an in depth history and physical is of utmost importance when evaluating child with recurrent right lower quadrant abdominal pain. In our study we used physical finding such as pain at McBurney's point during the time of an episode, pain that caused the child to squirm, rather than lay still as seen in acute appendicitis, to support our diagnosis. Patients who presented with peritoneal signs were excluded. Supportive history were; greater than three episodes for three or more weeks, patients were asymptomatic between episodes, aggravated or precipitated by eating, or physical activity. In Stevensons' series postprandial exacerbation of pain was felt to be of significance because it identifies the GI tract as the source of discomfort [4]. There are three main reasons that lead to confusion on this subject which are patients can be symptom free thus having a normal physical exam, the difficulty in imaging the appendix, and negative laboratory workup therefore making a diagnosis more difficult because of lack of physical evidence.

The etiologies: fecalith, inspissated stool, parasites, foreign matter, anatomical kinks, adhesions, and lymphoid hyperplasia are well described in the literature. The presence of a fecalith or inspissated cast of stool within the appendiceal lumen consistently remain common causes.
In a study of 71,000 human appendix done by Collins, a $44.3 \%$ incidence of fecaliths was noted [7]. Felson also reported a 33\% incidence of fecaliths in acutely inflamed appendix and $2.7 \%$ in normal appendix [8]. Schisgall, reports and incidence of $12.9 \%$ who had a normal appendix post appendectomy [3]. Our data states that imaging in patients with appendiceal colic is often of little yield. Gorentstein and Schisgall alike, support the use of radiographic studies such a barium swallow with small bowel follow through or a barium enema [9]. Both studies support a relationship between appendiceal colic, filling defects of the appendix after 24 hours and the failure for the appendix to empty after 72 hours. It is unclear weather there is a correlation in size or anatomical orientation of the appendiceal orifice and the increase in incidence of fecalith obstruction or inspissated stool. In their studies the presence of a filling defect, failure of the appendix to fill, or failure of the appendix to empty were used as criteria for an elective appendectomy and recommend routine radiographic evaluation in patient with suspected appendiceal colic. A pattern of appediceal colic is seen in those with recurrent upper respiratory infection secondary to lymphoid hyperplasia and symptom usually regress with the resolution of the URI. Appendiceal colic secondary to obstruction via a foreign body or pinn worms are usually found in younger children or those with PICA.

In our experience we found it beneficial to use an algorithmic approach to diagnose and treat patients suspected of appendiceal colic. Patients presenting for evaluation of recurrent RLQ abdominal pain classic for appendicitis and demonstrating an increased CRP and WBC were treated with an open appendectomy. The diagnosis becomes a little less clear when they present with recurrent right lower quadrant pain without an increase in CRP and WBC. More often than not these patients arrived at our office after having a completely negative workup including imaging and blood test. For those whom where not radiographically evaluated we prefer and obtained an abdominal ultrasound. If the imaging was unrevealing for any other pathology we elected to observe these patients for up to three weeks. Patients need to be reevaluated within three weeks or at the time of a painful episode. When McBurneys point tenderness was present during a painful episode, painful episodes persisted for longer than three weeks, and all other differential diagnosis were tolled out we then proceeded with an elective appendectomy.

Although an organic cause of recurrent right lower can be diagnosed in less than ten percent of patients the concept of appendiceal colic remains controversial. This subject has been described by many different authors with no clear consensus. In our experience appendiceal colic is a true surgical entity for a small subset of patients who meet a defined set of criteria of RLQ pain with McBur- 


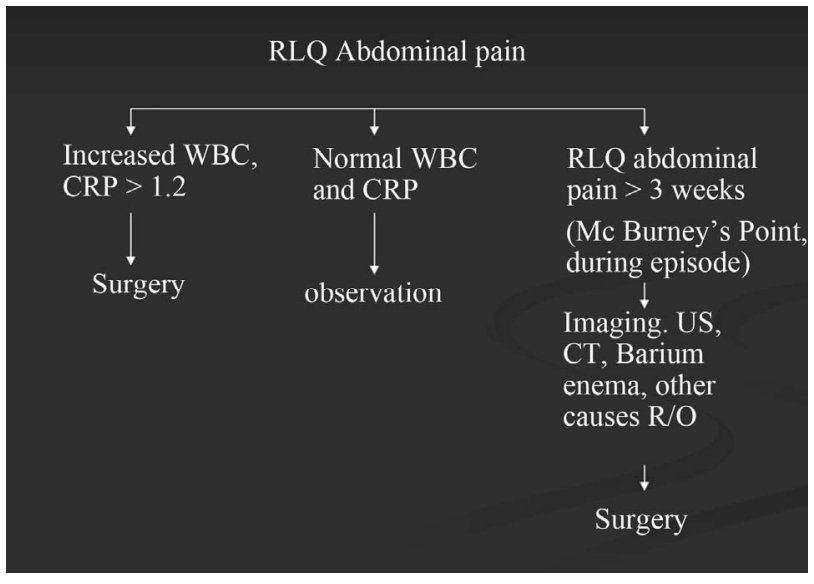

Figure 4. Workup of right lower quadrant abdominal pain.

ney's point tenderness during an episode, pain for more than three weeks and having greater than three episodes, and negative imaging and laboratory workup (Figure 4). It often presents as recurrent right lower quadrant abdominal pain secondary to a partial obstruction of the appendiceal lumen. An elective appendectomy has proven to curative in our experience.

\section{REFERENCES}

[1] D. E. Robertson, “Appendiceal Colic,” Canadian Medical
Association Journal, Vol. 38, No. 5, 1938, pp. 443-445.

[2] W. F. Bowers, "Appendicitis. With Special Reference to Pathogenesis, Bacteriology, and Healing," Archives of Surgery, Vol. 39, No. 3, 1939, pp. 362-422. doi:10.1001/archsurg.1939.01200150041003

[3] R. M. Schisgall, "Appendiceal Colic in Childhood, the Role of Inspissated Cast of Stool within the Appendix," Annals of Surgery, Vol. 192, No. 5, 1980, pp. 687-693. doi:10.1097/00000658-198019250-00016

[4] R. J. Stevenson, "Chronic Right-Lower-Quadrant Abdominal Pain: Is There a Role for Elective Appendectomy?" Journal of Pediatric Surgery, Vol. 34, No. 6, 1999, pp. 950-954. doi:10.1016/S0022-3468(99)90766-2

[5] P. Mattie, "Chronic and Recurrent Appendicitis Are Uncommon Entities and Often Misdiagnosed," Journal of the American of College of Surgeons, Vol. 178, No. 4, 1994, pp. 385-389.

[6] J. Apley, "The Child with Recurrent Abdominal Pain," Pediatric Clinics of North America, Vol. 14, No. 1, 1967, pp. 63-72.

[7] D. C. Collins, "71,000 Human Appendix Specimens. A Final Report, Summarizing Forty Years’ Study,” American Journal of Proctol, Vol. 14, 1963, pp. 365-381.

[8] B. Felson, “Appendiceal Calculi,” Surgery, Vol. 25, 1949, pp. 734-747.

[9] A. Gorenstein, "Appendiceal Colic in Children: A True Surgical Entity?” Journal of the American College of Surgeons, Vol. 182, No. 3, 1996, pp. 246-250. 